\title{
Thresholds and Upper Limits of Training-Induced Cardiovascular, Respiratory, Immune, and Musculoskeletal Adaptations in Able-Bodied People and Neurologically Impaired Patients
}

\author{
Pierre A Guertin* \\ Department of Psychiatry and Neurosciences, Faculty of Medicine, Laval University, Laval University Medical Center, Canada
}

*Corresponding author: Pierre A Guertin, Department of Psychiatry and Neurosciences, Faculty of Medicine, Laval University, Laval University Medical Center, Canada

\begin{abstract}
The benefits of exercising are well-recognized-experts and governmental health agencies agree that regular endurance training generally reduces the risks of metabolic and cardiovascular diseases. In turn, the consequences of a sedentary lifestyle and physical inactivity caused by disease or trauma are also well-documented- e.g., walking or being moderately active physically less than 30 minutes per day generally increases the risks of developing obesity, infections, osteoporosis, metabolic disorders, cardiovascular problems, and dyslipidemia in able-bodied persons. However, the threshold levels of exercising for meaningful health benefits in people suffering from paralysis or the upper limits of endurance training beyond which, injuries, sequelae, and long-term secondary problems that may be induced in able-bodied and people with disabilities remain unclear. This said, it is becoming increasingly clear that significant health problems including severe stress injuries and sudden heart failures may be experienced during long-distance running - e.g., marathons, triathlons, and ultra-triathlons. This review summarizes data on injuries and fatal events associated with endurance exercise training. Impacts on the immune, cardiovascular, respiratory, and musculoskeletal systems in disabled and nondisabled individuals are also discussed.
\end{abstract}

Keywords: Spinal Cord Injury; Performances; Marathon; Iron Man; Tendinitis; Cardiac Arrest; Infections; Stressed Fractures; Overtraining

\section{Introduction}

The Marathon as we know it today exists since the late 19th century. It is essentially a long-distance race of 42 kilometers (42.195 kilometers) usually performed on the road. It can be completed by running or running/walking for able-bodied persons as well as by wheel chairing for disabled individuals. About 800 marathons are held each year worldwide. Most of the competitors are recreational athletes. Progressively, over the years, longdistance races have become even harder. In the late 80s and early 90s - e.g., ultra-marathons (e.g., 100 kilometers), triathlons and Ironman races (1.5 kilometer swim, 40 kilometer bike ride and 10 kilometer run), or ultra-triathlons (e.g., the Uber Man created in 2017 is composed of a 33 kilometer swim, a 643 kilometer bike ride, and a 217 kilometer run).

Until recently, it was generally believed that marathon runners were not particularly at risks for cardiovascular and respiratory problems since cardiac deaths were reported to be almost 'nonsignificant' Maron et al. [1]. However, as exciting and healthy as it may look like, marathons can alter the function of several biological systems and organs. It is not uncommon indeed for runners to experience acute musculoskeletal injuries, gastrointestinal problems, and life-threatening hyponatremia Sanchez et al. [2]. The latter is increasingly encountered due to overhydration with hypotonic fluids. 
The recent enthusiasm for extreme events such as triathlons, ultra-marathons, and ultra-triathlons has enabled more data to be generated and new findings to be made. Among them, the number of injuries and fatal deaths has increased significantly.

For instance, sudden cardiac death (SCD) is described as an event that is non-traumatic, non-violent, unexpected and resulting from sudden cardiac arrest within six hours of previously witnessed normal health.

Prolonged exercise can trigger unheralded ventricular arrhythmias and SCD in individuals without no previous heart problems. In apparently healthy long-distance runners, the incidence of SCD ranges may reach 1:15,000 per year. SCD is more frequent in male and black athletes as well as in older individuals Kim et al., Ghio et al. [3, 4].

The heart of lifelong male endurance athletes generally contains more plaque or other signs of heart problems (e.g., myocardial fibrosis, late gadolinium enhancement), such as scarring and inflammation, than the hearts of less active men of the same age.

Physiological adaptations to triathlon and ultramarathon training include increased left ventricular cavity size and/or wall thickness. However, after a competition, cardiac problems associated with heart muscle fatigue may be found. Musculoskeletal adaptations to proper training include increased muscle respiratory capacity and substrate utilization modifications. The musculoskeletal system is the site of most injuries for those performing high endurance exercise training - i.e., non-traumatic overuse injuries are found in 80 to $85 \%$ of the musculoskeletal injuries. An imbalance caused by overly intensive training and inadequate recovery leads to a breakdown in tissue reparative mechanisms and eventually to overuse injuries Cosca et al. [5].

Overuse injuries associated with inflammatory mechanisms are also often found. They include patellofemoral pain syndrome, iliotibial band friction syndrome, medial tibial stress syndrome, Achilles tendinopathy, plantar fasciitis, and lower extremity stress fractures. Moreover, endurance athletes are more at risk for exercise-associated medical conditions, including exerciseinduced asthma, exercise-associated collapse, and overtraining syndrome. Exercise-induced immunodepression and exaggerated inflammatory response Castell et al. [6] affecting the upper respiratory tract and the gastrointestinal system may also be found - e.g., intensive triathlon training induces low peripheral CD34+ stem cells Phillip et al. [7]. Endurance athletes often quickly develop exercise-induced bronchoconstriction, two- or threefold greater (incidence) than the rate of asthma in the general population Knopfli et al. [8]. Overall, up to $70 \%$ of recreational and competitive runners sustain overuse injuries during any 12-month period (Australian Sports Commission's 2006).
It was clearly demonstrated recently by a large-scale study with more than 9 million participants that SCD affected that is 135 people Harris et al. [9]. The victims were 47 years of age on average, and 85 percent were male. Overall, 90 deaths and cardiac arrests occurred during the swimming portion of races. The incidence of cardiovascular events was strikingly lower in female triathletes, 3.5-fold less than in men. Several experts believe that they may constitute an underestimation of the reality since only data from the finishers were considered. Yet, it shows that deaths and SCD during long-distance events are not rare Harris et al. [9].

In people living with disabilities such as with paralysis caused by a spinal cord injury (SCI), the consequences of physical inactivity are also well-known-within a few weeks to a few months post-injury, several organs and systems including bones, muscles, immune cells, guts, skin, brain, blood cells, and heart, generally undergo significant dysregulations that lead to the development of chronic diseases and polymorbidity problems. Many of those socalled 'secondary complications' are not associated with the injury per se but, instead, with the main consequence of physical inactivity mainly in completely paralyzed patients Wilson et al. [10].

Extensive work, mainly from Bauman, has characterized the extent after SCI of lean body mass loss, bone loss, fractures, adiposity increase, obesity, anabolic hormone decrease, insulin resistance, hypertension, cholesterol, incidence of type II diabetes, cardiovascular problems, anxiety and depression Bauman et al. ; Bauman [11, 12]; Battalio et al. [13]. Immunodeficiency and its role in frequent infections (e.g., UTIs, skin sores, septicaemia, pneumonia) and premature death has also been established Brommer et al. [14]. Overall, 19 classes of drugs and 300 different compounds are used against chronic comorbidities post-SCI Rouleau et al. [15].

Although it has clearly been shown in able-bodied persons with a sedentary lifestyle that significant exercise training such as active walking more than 30 minutes per day can prevent or reverse these problems, comparable effects for those with SCI are incompletely characterized Krassioukov et al. [16]. Yet, it was shown in paraplegic mice treated with Spinalon, an experimental tritherapy that activates during 30 minutes the spinal locomotor networks, that an increase of muscle mass (25\%), femoral bone mineral density (10\%), red blood cells/haematocrit levels (10\%), and lymphocyte counts (25\%) occurs after just a few weeks of metabolically challenging exercising on a treadmill induced pharmacologically Guertin et al. [17]; Ung et al. [18]. Preliminary evidence of efficacy in patients was recently reported also Radhakrishna et al. [19].

In patients with less severe injuries (incomplete SCIs), specialized rehabilitation approaches such as body-weightsupported treadmill training (BWSTT, Barbeau et al. [20]; Wernig et al. [21] and functional electrical stimulation (FES) biking have 
been shown to improve voluntary walking capabilities via longlasting effects on spinal plasticity as well as cardiovascular and cardiometabolic functions, to some extent Coupaud et al. [22]; Kapadia et al. [23]; Graham et al. [24].

However, for those with severe SCIs, BWSTT and FES used separately do not generally induce comparable health benefits although small effects can occasionally be found Frotzler et al. [25]. For instance, epidural stimulation (ES) + BWSTT was recently shown in 4 volunteers with AIS-B injuries to increase lean body mass, decrease body fat, and improve android/gynoid ratio, resting metabolic rate and VO2 max Terson de Paleville et al. [26]. BWSTT+exoskeletons (EXs) in 5 volunteers with SCI provided preliminary evidence of moderate benefits on VO2 max and peak heart rates Evans et al. [27].

All in all, it appears that medical devices used alone or with BWSTT can lead, to some extent, to detectable but moderate benefits essentially in individuals with incomplete SCIs. However, with complete SCIs, these approaches are promising, but not yet ideally suited for significant metabolic outcomes Ditors et al. [28]; Ter Woerds et al. [29]; van Duijnhoven et al. [30] suggesting that other combinatorial approaches such as drug + device need to be explored Gerasimenko et al. [31]; Freyvert et al. [32]; Bloch [33].

\section{Concluding Remarks}

The long-term consequences on health degradation and system/organ dysregulations of physical inactivity or of a sedentary lifestyle are undisputable. In able-bodied persons, walking daily more than 30 minutes is generally recognized as the threshold level for preventing those complications. However, for disabled and particularly for wheelchair-bound persons -e.g., with a severe SCI- the threshold for preventing polymorbidities, systemic dysregulations, and overmedication problems remains unclear and is currently being explored. New solutions using pharmacological, robotic, and electrical approaches as combinatorial therapies are promising. In turn, for able-bodied and wheelchair athletes capable of performing long-distance competitions such as marathons, ultra-marathons, and other comparable events, the risk of deaths is significant but relatively low. In turn, the incidence of myocardial fibrosis and other cardiac problems is high over time as well as the incidence of overuse problems that is by far the more important type of injuries.

\section{References}

1. Maron BJ, Shirani J, Poliac LC, Mathenge R, Roberts WC, et al. (1996) Sudden death in young competitive athletes : clinical, demographic, and pathological profiles. JAMA 276(3): 199-204.

2. Sanchez LD, Corwell B, Berkoff D (2006) Medical problems of marathon runners. Am J Emerg Med 24(5): 608-615.
3. Kim J H, Malhotra R, Chiampas G, et al. (2012) Cardiac arrest during long-distance running races. N Engl J Med 366: 130-140.

4. Ghio FE, Pieri M, Agracheva A, Melisurgo G, Ponti A, et al. (2012) Sudden cardiac arrest in a marathon runner. A case report. HSR Proc Intensive Care Cardiovasc Anesth 4(2): 130-132.

5. Cosca DD, Navazio F (2007) Common problems in endurance athletes. Am Fam Physician 76(2): 237-244.

6. Castell LM, Nieman DC, Bermon S, Peeling P (2019) Exercise-induced illness and inflammation : can immunonutrition and iron help. International Sport Nutrition and Exercise Metabolism 29(2): 181-188.

7. Philip P, Bermon S (2003) Intensive triathtlon training induces low peripheral CD34+ stem cells. Br J Haematol 120(5): 914-915.

8. Knopfli BH, Luke-Zeitoun M, von Duvillard SP, Adrian Burki, Christian Bachlechner, et al. (2007) High incidence of exercise-induced bronchoconstriction in triathletes of the Swiss National Team. Br J Sports Med 41(8): 486-491.

9. Harris KM, Creswell LL, Haas TS, Taylor Thomas, Monica Tung, et al. (2017) Death and cardiac arrest in US triathlon participants, 1985 to 2016 : a case series. Ann Inter Med 167(8): 529-535.

10. Wilson M, O'Hanlon R, Prassad S, A Deighan, P Macmillan, et al. (2011) Diverse patterns of myocardial fibrosis in lifelong, veteran endurance athletes. J Appl Physiol 110(6): 1622-1626.

11. Bauman, Spungen AM, Adkins RH, Kemp BJ, et al. (1999) Metabolic and endocrine changes in persons aging with spinal cord injury. Assist Technol 11(2): 88-96.

12. Bauman, Spungen (2000) Metabolic changes in persons after spinal cord injury.Phys Med Rehabil Clin N Am 11(1):109-140.

13. Battalio, Mari Glette, Kevin N Alschuler, Mark P Jensen et al. (2018) Anxiety, depression, and function in individuals with chronic physical conditions: a longitudinal analysis. Rehabil Psychol 63(4): 532-541.

14. Brommer, Odilo Engel, Marcel A Kopp, Ralf Watzlawick, Susanne Müller, et al. (2016) SCI-induced immune deficiency syndrome enhances infection susceptibility dependent on lesion level. Brain 139(pt 3): 692707.

15. Rouleau, Guertin (2011) Traumatic and non-traumatic SCI patients in Quebec, Canada. Part 3 : pharmacological characteristics. Spinal Cord 49(2): 186-195.

16. Krassioukov, Katharine D Currie, Michèle Hubli, Tom E Nightingale, Abdullah A Alrashidi et al. (2019) Effects of exercise interventions on cardiovascular health in individuals with chronic, motor complete SCI: protocol for a randomized controlled trial. BMJ Open 9(1): e023540.

17. Guertin, Ung, Rouleau (2011) Effects on locomotion, muscle, bone and blood induced by a combination therapy eliciting weight-bearing stepping in non-assisted Tx mice. Neurorehabil Neural Repair 25(3): 234-242.

18. Ung, Rouleau, Guertin (2012) Functional and physiological effects of treadmill training induced by buspirone, carbidopa, and L-Dopa in clenbuterol-treated paraplegic mice Neurorehabil Neural Repair 26(4): 385-394.

19. Radhakrishna M, Steuer I, Prince F, Roberts M, Mongeon D, Kia M, Dyck S, Matte G, Vaillancourt M, Guertin, P.A. (2017) Double-blind, placebocontrolled, randomized phase I/IIa study (safety and efficacy) with buspirone/levodopa/carbidopa (Spinalon) in subjects with complete AIS A or motor-complete AIS B spinal cord injury. Current Pharmaceutical Design 23(12): 1789-1804. 
20. Barbeau, M Wainberg, L Finch (1987) Description and application of a system for locomotor rehabilitation. Med Biol Eng Comput 25(3): 341 344 .

21. Wernig, Muller (1992) Laufband locomotion with BWS inproved walking in persons with severe SCI. Paraplegia 30(4): 229-238.

22. Coupaud, H Gollee, K J Hunt, M H Fraser, D B Allan, et al. (2008) Armcranking exercise assisted by FES in C6 tetraplegia: a pilot study. Technol Health Care 16(6): 415-427.

23. Kapadia, Kei Masani, B Catharine Craven, Lora M Giangregorio, Sander L Hitzig, et al. (2014) A randomized trial of FES stimulation for walking in incomplete SCI. Effects on walking competency. J Spinal Cord Med 37(5): 511-524.

24. Graham, Ceren Yarar-Fisher, Jia Li, Kevin M McCully, James H Rimmer et al. (2019) Effects of high-intensity interval training versus moderateintensity training on cardiometabolic health markers in individuals with SCI: a pilot study. Top Spinal Cord Inj Rehabil 25(3): 248-259.

25. Frotzler A, Sylvie Coupaud, Claudio Perret, Tanja H Kakebeeke, Kenneth J Hunt, et al. (2008) High-volume FES-cycling partially reverses bone loss in people with chronic SCI. Bone 43(1): 169-176.

26. Terson de Paleville, Susan J Harkema, Claudia A Angeli (2019) Epidural stimulation with locomotor training improves body composition in individuals with cervical or upper thoracic motor complete SCI : a series of case studies. J Spinal Cord Med 42(1): 32-38.
27. Evans, Brooks Wingo, Elizabeth Sasso, Audrey Hicks, Ashraf S Gorgey, et al. (2015) Exercise recommandations and considerations for persons with SCI. Arch Phys Med Rehabil 96(9): 1749-1750.

28. Ditor, M J Macdonald, M V Kamath, J Bugaresti, M Adams, et al. (2005) The effects of BWSTT on cardiovascular regulation in individuals with motor-complete SCI. Spinal cord 43(11): 664-673.

29. Ter Woerds, Patricia C E De Groot, Dirk H J M van Kuppevelt, Maria T E Hopman (2006) Passive leg movements and passive cycling do not alter arterial leg blood flow in subjects with SCI. Phys Ther 86(5): 636-645.

30. Van Duijnhoven, Evelyne Hesse, Thomas Janssen, Will Wodzig, Peter Scheffer et al. (2008) Impact of exercise training on oxidative stress in individuals with a SCI. Eur J Appl Physiol 109(6): 1059-1066.

31. Gerasimenko, Ruslan Gorodnichev, Aleksandr Puhov, Tatiana Moshonkina, Aleksandr Savochin et al. (2015) Initiation and modulation of locomotor circuitry output with multisite transcutaneous electrical stimulation of the spinal cord in noninjured humans. J Neurophysiol 113(3): 834-842.

32. Freyvert Y, Au Yong N, Morikawa E, Sharon Zdunowski, Melanie E Sarino, et al. (2018) Engaging cervical spinal circuitry with non-invasive spinal stimulation and buspirone to restore hand function in chronic motor complete patients. Sci Rep 8(1): 15546.

33. Bloch (2020) STIMO Epidural electrical stimulation EES with robotassisted rehabilitation in patients with SCI. US National Library of Medicine, clinicaltrials.gov.

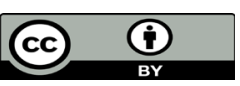

This work is licensed under Creative Commons Attribution 4.0 License

To Submit Your Article Click Here: Submit Article

DOI: 10.32474/ACR.2020.03.000157

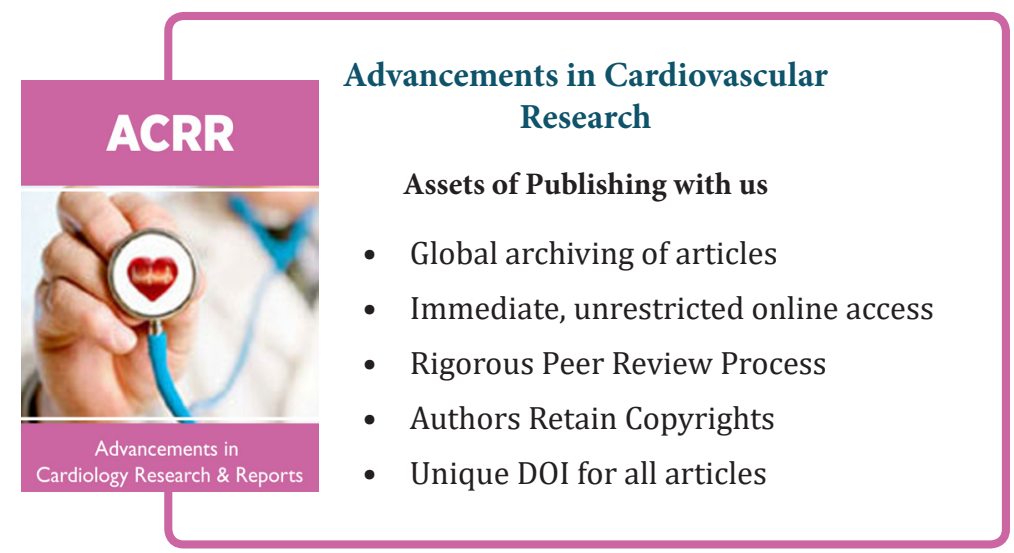

